\title{
Clinical efficacy of low dose recombinant tissue- type plasminogen activator for the treatment of acute intermediate-risk pulmonary embolism
}

Ling-Yun Zhang, MD, Bao-An Gao, PhD, Zhu Jin, PhD, Guang-Ming Xiang, MD, Zheng Gong, PhD, Ting-Ting Zhang, MD, Hong-Fang Lu, MD, Yong-Quan Wang, MD, Yuan Gong, MD, Cheng Lu, MD, Wei-Ling Huang, MD.

\section{ABSTRACT}

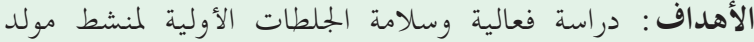

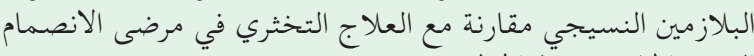

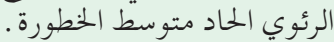

$$
\begin{aligned}
& \text { الطريقة: بتقسيم } 66 \text { مريض يعاني من الانصمام الرئوي الحاد }
\end{aligned}
$$

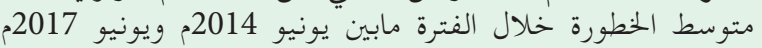

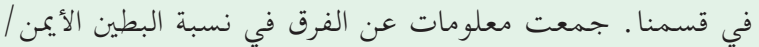

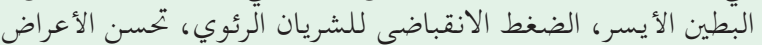

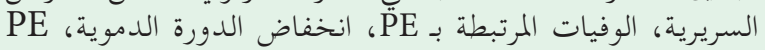

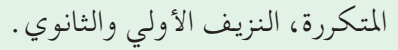

النتائج : أنخفض متوسط PASP من 12.2

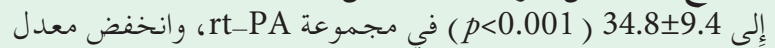

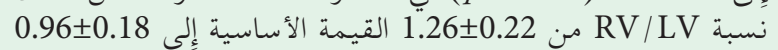

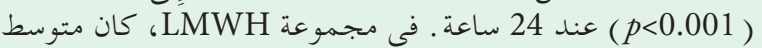

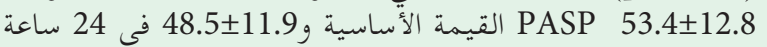

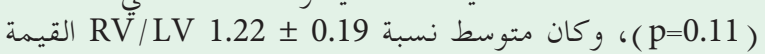

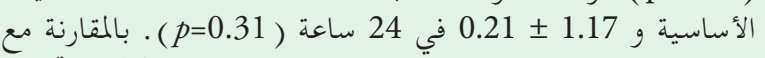

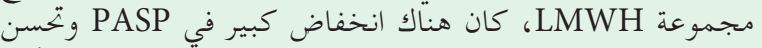

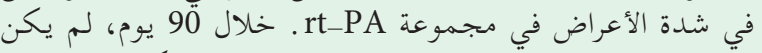

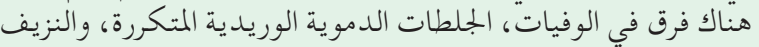

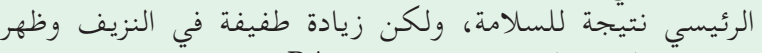

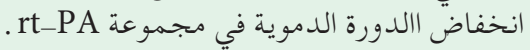

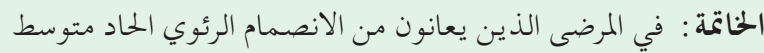

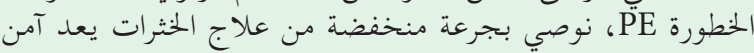

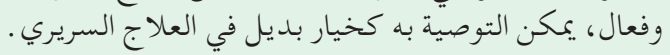

Objectives: To investigate the efficacy and safety of initial thrombolysis by recombinant tissue-type plasminogen activator (rt-PA) in compared with anticoagulant therapy in patients with acute intermediate-risk pulmonary embolism (PE).

Methods: Sixty-six patients with acute intermediaterisk $\mathrm{PE}$ were randomly assigned to receive rt-PA or
LMWH between June 2014 and June 2017 in our department. We obtained information regarding the difference in the right ventricle/left ventricle (RV/ LV) ratio, pulmonary artery systolic pressure (PASP), clinical symptoms improvement, PE-related mortality, hemodynamic decompensation, recurrent PE, and major and minor bleeding.

Results: In the rt-PA group, the mean PASP was reduced from $52.0 \pm 12.2$ at baseline to $34.8 \pm 9.4(p<0.001)$ and the mean $\mathrm{RV} / \mathrm{LV}$ ratio was reduced from $1.26 \pm 0.22$ at baseline to $0.96 \pm 0.18 \quad(p<0.001)$ at 24 hours. In the LMWH group, the mean PASP was $53.4 \pm 12.8$ at baseline and $48.5 \pm 11.9$ at 24 hours $(p=0.11)$, and the mean $\mathrm{RV} / \mathrm{LV}$ ratio was $1.22 \pm 0.19$ at baseline and $1.17 \pm 0.21$ at 24 hours $(p=0.31)$. In comparison with the LMWH group, there was a significant reduction in PASP and an improvement in the symptom severity in the rt-PA group. At 90 days, there was no difference in mortality, recurrent venous thromboembolism and major bleeding as a safety outcome, but increased minor bleeding and decreased hemodynamic decompensation occurred in the rt-PA group.

Conclusions: In patients with acute intermediate-risk $\mathrm{PE}$, low dose thrombolytic therapy is considered safe and effective, it can be recommended as an alternative option in clinical treatment.

Saudi Med J 2018; Vol. 39 (11): 1090-1095 doi: $10.15537 /$ smj.2018.11.22717

From the Department of Respiratory and Critical Care Medicine (Zhang, Gao, Jin, Xiang, Gong, Zhang, Lu, Wang, Gong), and from the Department of Cardiology (Lu, Huang), The First College of Clinical Medical Science of China Three Gorges University \& Yichang Central People's Hospital, Yichang, China.

Received 16th August 2018. Accepted 10th October 2018.

Address correspondence and reprint request to: Dr. Bao-an Gao Department of Respiratory and Critical Care Medicine, The First College of Clinical Medical Science of China Three Gorges University \& Yichang Central People’s Hospital, Yichang, China. E-mail: 459118966@qq.com ORCID ID: orcid.org/0000-0001-9421-1156 
A cute pulmonary embolism (PE) is a potentially life-threatening disease that can result in significant morbidity and mortality for patients. Thrombolytic therapy is currently recommended for the treatment of high-risk PE. In the recent ESC guidelines, it also is considered for acute intermediate-risk PE patients with right-ventricular (RV) dysfunction or myocardial injury. ${ }^{1,2}$ In clinical trials, thrombolysis has been shown to improve hemodynamic parameters, prevent recurrent $\mathrm{PE}$, and reduce mortality. ${ }^{3,4}$ However, it has a serious risk of intracranial hemorrhage which was reported to be $0.7 \%$ to $6.4 \%$ in patients receiving thrombolysis. As a result, many clinicians hesitate in ordering thrombolytic therapy, and the benefit of extending thrombolytic treatment to acute intermediate-risk PE needs to be weighed against potential disadvantages in terms of bleeding risk. Additionally, how to choose the appropriate form of administration of thrombolysis and what the optimal dose of thrombolysis should be was investigated.

Recombinant tissue-type plasminogen activator (rt-PA) is currently one of the most widely used and the most effective thrombolytic agents for PE, it is very important to use optimal dose because of its dosedependent risk of bleeding. In the traditional systemic thrombolytic therapy, rt-PA was given at a full dose of $100 \mathrm{mg}$ during a 2-hour infusion. Although effective, it is associated with an unacceptable high bleeding complication rate. Many patients are unwilling to accept this form of treatment in the clinic. In recent studies, low dose thrombolysis by rt-PA has been employed in the treatment of both massive and submassive PE. The data showed that it has far fewer bleeding complications than full dose systemic thrombolysis yet exhibits equal efficacy. ${ }^{5-7}$ However, the optimal dose of rt-PA has not been confirmed, and appropriate patients should be chosen.

Methods. Patients and study design. From June 2014 to June 2017, a total of 76 acute intermediaterisk PE patients were screened in the Department of Respiratory Medicine, The First College of Clinical Medical Science of China Three Gorges University \& Yichang Central People's Hospital, Yichang, China; 10 patients were ineligible and were excluded according to the exclusion criteria. Finally, 66 patients aged 18 to

Disclosure. Authors have no conflict of interests, and the work was not supported or funded by any drug company.

80 years with acute intermediate-risk PE from Yichang Central People's Hospital in China were randomly assigned by envelopes to receive rt-PA at a dose of $30 \mathrm{mg}$ over 2 hours $(\mathrm{n}=33)$ with concomitant lowmolecular-weight heparin (LMWH) anticoagulation or LMWH anticoagulation alone $(\mathrm{n}=33)$ (Clinical Trial Identifier:2014A06035). All diagnoses were confirmed by the presence of an intraluminal filling defect on spiral computed tomographic pulmonary angiography (CTPA) examination. Echocardiography was performed prior to initiation of thrombolysis and within 24 hours post-thrombolysis in all the patients. Pulmonary artery systolic pressure (PASP) was estimated from the tricuspid valve regurgitant jet velocity using a modified Bernoulli's equation. Pulmonary hypertension (PH) was defined as a PASP of $\geq 30 \mathrm{~mm} \mathrm{Hg}$, as determined by echocardiography. Right ventricular dysfunction was defined as a right ventricle/left ventricle (RV/LV) ratio of $>0.9$ by echocardiography. A positive troponin test was defined as an elevation of troponin I $(>0.04 \mu \mathrm{g} / \mathrm{L})$. Hemodynamic decompensation was defined by the need for cardiopulmonary resuscitation, a drop in SBP by $\geq 40 \mathrm{~mm} \mathrm{Hg}$ for $\geq 15 \mathrm{~min}$ or $\mathrm{SBP}<90 \mathrm{~mm} \mathrm{Hg}$ for $\geq 15 \mathrm{~min}$ in accompanied with hypoperfusion of end-organ, or the need for vasopressors. The patients were asked about their subjective perception of severity of symptoms before and after treatment on an arbitrary scale from 1-10, with 1 as having no symptoms and 10 with severe and unbearable symptoms (mainly chest tightness and dyspnea). The patients were evaluated daily while in the hospital. The follow-up was 3 months after discharge.

The inclusion criteria were acute symptomatic PE confirmed by CTPA with an embolus located in at least 1 main or proximal lower lobe pulmonary artery and RV/LV ratio $\geq 0.9$ obtained on echocardiographic examination. Exclusion criteria were age $<18$ or $>80$ years; index PE symptom duration >14 days; uncontrolled hypertension (SBP $>180 \mathrm{~mm} \mathrm{Hg}$ and/ or diastolic blood pressure (DBP) $>110 \mathrm{~mm} \mathrm{Hg}$ at presentation; SBP $<90 \mathrm{~mm} \mathrm{Hg}$ for more than $15 \mathrm{~min}$ at presentation, with or without signs of cardiogenic shock; known significant bleeding risk; active bleeding; known coagulation disorder; gastrointestinal bleeding within the preceding 3 months; history of any intracranial or intraspinal surgery or trauma or intracranial/ intraspinal bleeding; arteriovenous malformation, or aneurysm; major surgery, cataract surgery, trauma, obstetric delivery, cardiopulmonary resuscitation, or other invasive procedure < 10 days; pregnancy, lactation, or parturition $<30$ days; participation in any other investigational drug or device study; life expectancy $<3$ months; and inability to comply with study assessments. 
The study is according to principles of Helsinki Declaration. It was approved by the Ethics Committees of the Institutions, and written informed consent was obtained from all of the patients.

Treatment regimen. Eligible subjects were randomly assigned to receive either $30 \mathrm{mg} \mathrm{rt}-\mathrm{PA}$ in continuous intravenous infusion for 2 hours with concomitant LMWH anticoagulation (rt-PA Group, $\mathrm{n}=33$ ) or LMWH anticoagulation alone (LMWH Group, $n=33$ ). We used rt-PA from Boehringer Ingelheim Pharma of Germany. The activated partial thromboplastin time was determined after the rt-PA infusion. If the value was less than 80 seconds, a subcutaneous injection of LMWH (enoxaparin, $1 \mathrm{mg} / \mathrm{kg}$ ) was given every 12 hours. Overlapping oral anticoagulant therapy (warfarin) was started from day one after enoxaparin injection. Enoxaparin was stopped after the INR was stabilized at 2.0 to 3.0 for at least 2 days. Warfarin was continuously used for at least 3 months, and subsequent doses were adjusted to maintain the INR within the 2.0 to 3.0 range, targeting a value of 2.5.

Study outcomes. Symptoms and signs of PE in all patients were monitored. The patients were evaluated for changes in the RV/LV ratio, PASP, and subjective improvement before rt-PA infusion, and the evaluation was repeated in all patients at 24 hours after rt-PA treatment. The main outcome measure is PASP. Bleeding, mortality, hemodynamic decompensation and recurrent venous thromboembolism during hospitalization and at follow-up were also evaluated.
Statistical analysis. Statistical analyses were performed with SPSS version 21 (IBM Corp., Armonk, $\mathrm{NY}$ ). Continuous variables were expressed as the mean \pm standard deviation, whereas categorical variables were given as numbers (percentages). A comparison between the 2 groups was made using an unpaired test for continuous variables and a Chi-squared or Fisher's exact test for categorical variables (2-tailed). A $p$-value 0.05 was considered statistically significant.

Results. Baseline clinical characteristics. Among the 66 patients, the mean age was $59.0 \pm 12.2$ years, and $48 \%$ were men. The most frequent comorbidities and risk factors were deep venous thrombosis (53\%), systemic hypertension $(42 \%)$, smoking $(24 \%)$, and coronary artery disease (14\%), with no difference between the study groups. Baseline vital parameters, including BMI, systemic arterial pressure, heart and respiratory rates, were similar between the groups, as well as laboratory findings of a positive troponin test (Table 1).

Efficacy outcomes. All patients had documented RV dysfunction on echocardiography and subjective perception of severity of symptoms. In the rt-PA group, the mean PASP was reduced from $52.0 \pm 12.2$ at baseline to $34.8 \pm 9.4(p<0.001)$ at 24 hours, and the mean $\mathrm{RV} / \mathrm{LV}$ ratio was reduced from $1.26 \pm 0.22$ at baseline to $0.96 \pm 0.18(p<0.001)$ at 24 hours. In the LMWH group, the mean PASP was $53.4 \pm 12.8$ at baseline and $48.5 \pm 11.9$ at 24 hours $(p=0.11)$. The mean $\mathrm{RV} / \mathrm{LV}$ ratio was $1.22 \pm 0.19$ at baseline and $1.17 \pm 0.21$ at 24 hours

Table 1 - Baseline clinical characteristics.

\begin{tabular}{|c|c|c|c|}
\hline Variables & \multicolumn{2}{|c|}{ rt-PA $30 \mathrm{mg} / 2$ hours } & LMWH $(\mathrm{n}=33)$ \\
\hline Age, years & $60.5 \pm 12.8$ & $58.6 \pm 11.4$ & 0.52 \\
\hline Men, n (\%) & $18(55)$ & $14(42)$ & 0.32 \\
\hline BMI, $\mathrm{kg} / \mathrm{m}^{2}$ & $24.5 \pm 3.4$ & $25.8 \pm 3.6$ & 0.13 \\
\hline Systolic arterial pressure, $\mathrm{mm} \mathrm{Hg}$ & $122.3 \pm 20.9$ & $120.2 \pm 18.7$ & 0.66 \\
\hline Diastolic arterial pressure, $\mathrm{mm} \mathrm{Hg}$ & $78.6 \pm 14.2$ & $75.2 \pm 12.8$ & 0.31 \\
\hline Heart rate, bpm & $85.0 \pm 17.4$ & $82.9 \pm 15.7$ & 0.60 \\
\hline Respiratory rate, per minute & $21.8 \pm 4.0$ & $23.4 \pm 4.3$ & 0.12 \\
\hline Current smoker, n (\%) & $9(27)$ & $7(21)$ & 0.57 \\
\hline Systemic hypertension, n (\%) & $16(48)$ & $12(36)$ & 0.32 \\
\hline Diabetes mellitus, n (\%) & $2(6)$ & 1 (3) & 1.00 \\
\hline Congestive heart failure, n (\%) & $3(9)$ & $2(6)$ & 1.00 \\
\hline Coronary artery disease, $\mathrm{n}(\%)$ & $5(15)$ & $4(12)$ & 1.00 \\
\hline COPD, n (\%) & $3(9)$ & $5(15)$ & 0.70 \\
\hline Cancer, n (\%) & $2(6)$ & $3(9)$ & 1.00 \\
\hline $\begin{array}{l}\text { Prior venous thromboembolism, } \\
\mathrm{n}(\%)\end{array}$ & $4(12)$ & $3(9)$ & 1.00 \\
\hline $\begin{array}{l}\text { Concomitant deep venous } \\
\text { thrombosis, } \mathrm{n}(\%)\end{array}$ & $19(57)$ & $16(48)$ & 0.46 \\
\hline Troponin test positive, $\mathrm{n}(\%)$ & $11(33)$ & $12(36)$ & 0.80 \\
\hline
\end{tabular}


Table 2 - Changes in echocardiographic and clinical parameters at 24 hours.

\begin{tabular}{|c|c|c|c|c|c|c|c|c|c|}
\hline \multirow[t]{2}{*}{ Variables } & \multicolumn{3}{|c|}{ rt-PA $30 \mathrm{mg} / 2$ hours } & \multicolumn{3}{|c|}{ LMWH } & \multicolumn{3}{|c|}{$\begin{array}{c}\text { Difference } \\
\text { Baseline versus } 24 \text { hours }\end{array}$} \\
\hline & Baseline & 24 hours & $P$-value & Baseline & 24 hours & $P$-value & rt-PA & Anticoagulation & $P$-value \\
\hline PASP, $\mathrm{mm} \mathrm{Hg}$ & $52.0 \pm 12.2$ & $34.8 \pm 9.4$ & $<0.001$ & $53.4 \pm 12.8$ & $48.5 \pm 11.9$ & 0.11 & $17.0 \pm 10.2$ & $4.6 \pm 9.8$ & 0.001 \\
\hline $\mathrm{RV} / \mathrm{LV}$ & $1.26 \pm 0.22$ & $0.96 \pm 0.18$ & $<0.001$ & $1.22 \pm 0.19$ & $1.17 \pm 0.21$ & 0.31 & $0.31 \pm 0.18$ & $0.04 \pm 0.16$ & 0.001 \\
\hline Symptoms severity & $7.0 \pm 1.8$ & $1.2 \pm 0.6$ & $<0.001$ & $6.8 \pm 1.9$ & $5.9 \pm 1.7$ & 0.05 & $5.6 \pm 1.5$ & $1.0 \pm 0.4$ & 0.001 \\
\hline
\end{tabular}

Table 3 - Clinical outcome events during the 3-month follow up

\begin{tabular}{lccc}
\hline Variables & $\begin{array}{c}\text { rt-PA 30 } 30 / 2 \\
\mathbf{n}=33\end{array}$ & $\begin{array}{c}\text { LMWH } \\
\mathbf{n}=33\end{array}$ & $P$-value \\
\hline $\begin{array}{l}\text { Death } \\
\text { Hemodynamic }\end{array}$ & 0 & 0 & - \\
decompensation & 0 & $3(9)$ & 0.24 \\
$\begin{array}{l}\text { Recurrent PE } \\
\text { Major bleeding }\end{array}$ & $1(3)$ & $2(6)$ & 1.00 \\
Minor bleeding & 0 & 0 & - \\
\multicolumn{4}{c}{ LMWH - low-molecular-weight heparin, PE - } \\
\hline
\end{tabular}

$(p=0.31)$. The mean difference in PASP from baseline to 24 hours was $17.0 \pm 10.2$ versus $4.6 \pm 9.8(p<0.001)$, respectively. The mean difference in $\mathrm{RV} / \mathrm{LV}$ ratio from baseline to 24 hours was $0.31 \pm 0.18$ versus $0.04 \pm 0.16$ $(p<0.001)$, respectively. In comparison to the LMWH group, there was a significant reduction in PASP and an improvement in symptom severity in the rt-PA group (Table 2).

Safety outcomes. At 90 days, there were no deaths and no major bleeding complication episodes among the 66 patients. There were 3 episodes of hemodynamic decompensation and 2 episodes of recurrent VTE in the LMWH group and no episodes of hemodynamic decompensation and 1 episode of recurrent VTE in the rt-PA group. Catecholamines were administered to the 3 hemodynamic decompensation patients during hospitalization in the LMWH group. All 3 recurrent VTE episodes happened between 3 months after discharge, 2 patients developed deep venous thrombosis and 1 developed PE. At the 90-day visit, all patients were on anticoagulation therapy with vitamin $\mathrm{K}$ antagonists or LMWH. Overall, there were 9 minor bleeding episodes: 8 patients (24\%) in the rt-PA group (3 with transient hemoptysis without medical intervention, 2 with gingival bleeding and 3 with puncture point bleeding managed with manual compression) and 1 patient (3\%) in the LMWH group with subcutaneous hemorrhage. There were no serious adverse events related to the study treatment (Table 3).
Discussion The results indicated that low dose thrombolysis by rt-PA at $30 \mathrm{mg} / 2$ hours is safe and effective in patients with acute intermediate-risk PE. Compared with anticoagulation treatment alone, thrombolysis can improve right heart function and reduce pulmonary artery pressure through dissolving the thrombus in a short period of time, it can also reduce the risk of heart function failure compensation and avoid missing the optimal opportunity for treatment. In the long run, it can reduce the probability of the formation of chronic thromboembolic pulmonary hypertension and recurrent pulmonary embolism, even if it has similar results with anticoagulation in mortality. Additionally, low dose thrombolysis did not increase the risk of major bleeding and it was safe, effective and controllable even though the minor bleeding was increased. There are significant implications for this approach:

Although rt-PA at $100 \mathrm{mg} / 2$ hours has been shown to be effective and recommended in guidelines as the standard for PE thrombolysis, the optimal regimens are less defined. In the "real world", even when clearly indicated, its use has been tempered by fear of major bleeding and especially intracerebral hemorrhage (ICH), which has usually been shown to occur in approximately $2-3 \%$ of patients. ${ }^{8}$ Recently, several studies have shown a reduced risk of bleeding with reduction of the dose of rt-PA while imparting the same beneficial effects seen in full dose thrombolysis, such as a $50 \mathrm{mg} / 2 \mathrm{~h}$ regimen or lower. ${ }^{9-12}$ In percutaneous ultrasound accelerated thrombolysis therapy, the dose of rt-PA is very low. ${ }^{13}$ Ozcinar et $\mathrm{a}^{14}$ showed the efficacy and safety of ultrasound-accelerated catheter-directed thrombolysis in the treatment of massive and submassive PE by rt-PA at a dose of $21 \mathrm{mg}$. In fact, no ICH has been reported with low dose thrombolysis with a modified dose of concomitant parenteral anticoagulation. In our early experience, we have witnessed an even lower rate of 
bleeding with a $50 \mathrm{mg} / 2 \mathrm{~h}$ rt-PA compared to standard parenteral anticoagulation. To obtain a higher safety, we designed this study and further reduced the dose of rt-PA to $30 \mathrm{mg}$. Our main purpose was to reduce the earlier mortality of acute intermediate-risk PE through decreasing the clot burden by thrombolysis, rather than curing the pulmonary embolism completely. Therefore, if similar results are reproduced and confirmed in larger studies, a new consensus to for the treatment of acute intermediate-risk PE will be reached and it could be used to replace conventional therapy.

According to the latest ESC Guidelines, patients not suffering from shock or hypotension require further risk stratification through assessing PESI (or sPESI), RV function by echocardiography and cardiac troponin testing after a diagnosis of PE has been confirmed. The intermediate-risk group can be divided into intermediate-high-risk and intermediate- low-risk group. Systemic thrombolysis is not routinely recommended as primary treatment for patients with intermediate-high-risk PE but should be considered if clinical signs of hemodynamic decompensation appear. It may present with more significant differences if only the intermediate-high-risk patients alone are included. Importantly, if we start with a standardized low dose thrombolytic therapy in a timely manner, rather than waiting for hemodynamic decompensation to appear, the risk of adverse events will be significantly reduced, and patients will receive timely treatment.

In the present study, the efficacy of thrombolysis was mainly driven by the prevention of hemodynamic decompensation by improving right heart function and reducing PSAP. In addition, it is indirectly demonstrated by significant improvements in the patient's clinical symptoms. Three patients with hemodynamic decompensation in the LMWH group

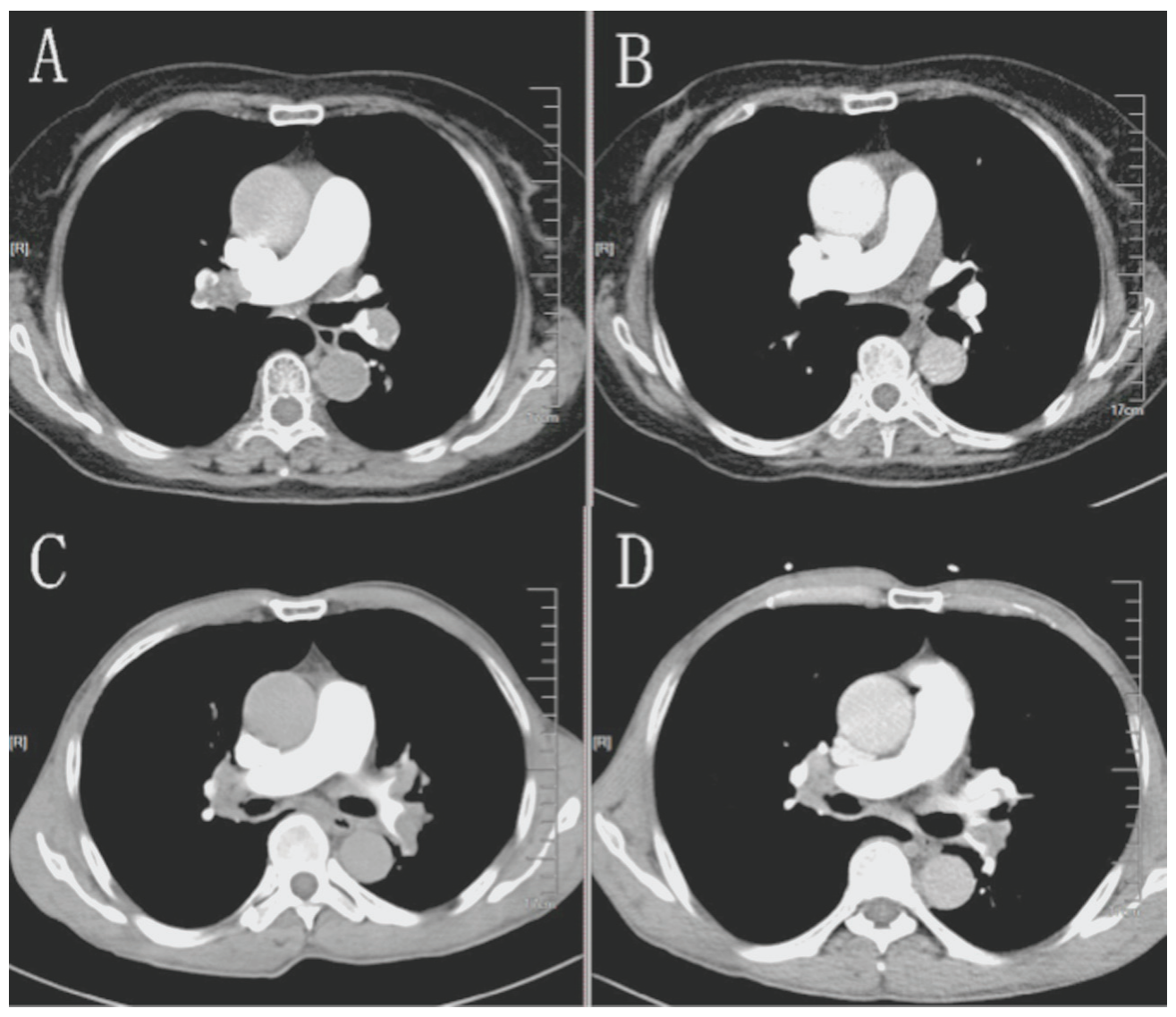

Figure 1 - A great resolution of the thrombus was confirmed by CTPA A) before and B) after the thrombolytic therapy (30 mg rt-PA IV over 2 hours) in a 75-year-old acute intermediate-risk PE woman. A little resolution of the thrombus was confirmed by CTPA C) before and D) after the thrombolytic therapy (30 mg rt-PA IV over 2 hours) in a 78-year-old acute intermediate-risk PE man. 
were given inotropic support and fortunately no deaths occurred. In the PEITHO study, the largest reported trial on thrombolytic therapy on submassive $\mathrm{PE}$ proposed that, if these patients had not been closely monitored and promptly treated when decompensation occurred, the prognosis would have been worse. ${ }^{15}$ Major bleeding, especially intracranial hemorrhage, has not been observed in the rt-PA group. The results of our study suggested that low-dose thrombolytic therapy is very safe and effective for acute intermediate-risk PE, especially for patients presenting with hemodynamic decompensation in the course of treatment.

Study limitations. There were a few limitations in our study. Our study was performed in a single center with a small sample size, there was no further refinement in the type of patient with acute PE at intermediate risk, and there was no effect when comparing higher and lower doses. Moreover, we found that clot burden changes vary greatly in different patients even when given the same dose of rt-PA before and after thrombolytic therapy (Figure 1). Therefore, there should be other factors that influenced the thrombolytic effects. Further investigations should be designed to confirm the optimal dosing of rt-PA and other affected factors for the treatment of PE; however, existing data suggest that low-dose rt-PA may be a successful treatment option for certain patients, particularly those at high risk of bleeding.

Acknowledgment. We would like to thank American Journal Experts for English language editing.

\section{References}

1. Jacobs B, Henke PK. Evidence-based therapies for pharmacologic prevention and treatment of acute deep vein thrombosis and pulmonary embolism. Surg Clin North Am 2018; 98: 239-253.

2. Konstantinides SV, Torbicki A, Agnelli G, Danchin N, Fitzmaurice D, Galiè N, et al. 2014 ESC guidelines on the diagnosis and management of acute pulmonary embolism. Eur Heart J 2014; 35: 3033-3073.

3. Sinha SK, Sachan M, Goel A, Singh K, Mishra V, Jha MJ, et al. Efficacy and safety of thrombolytic therapy in acute submassive pulmonary embolism: Follow-up study. J Clin Med Res 2017; 9: 163-169.
4. Chen H, Ren C, Chen H. Thrombolysis versus anticoagulation for the initial treatment of moderate pulmonary embolism: a meta-analysis of randomized controlled trials. Respir Care 2014; 59: 1880-1887.

5. Canan Hasanoğlu H, Hezer H, Karalezli A, Argüder E, Kiliç $\mathrm{H}$, Şentürk A, et al. Half-dose recombinant tissue plasminogen activator treatment in venous thromboembolism. J Investig Med 2014; 62: 71-77.

6. Sharifi M, Bay C, Schwartz F, Skrocki L. Safe-dose thrombolysis plus rivaroxaban for moderate and severe pulmonary embolism: drip,drug, and discharge. Clin Cardiol 2014; 37: 78-82.

7. Brandt K, McGinn K, Quedado J. Low-dose systemic alteplase (tPA) for the treatment of pulmonary embolism. Ann Pharmacother 2015; 49: 818-824.

8. Sista AK, Horowitz JM, Goldhaber SZ. Four key questions surrounding thrombolytic therapy for submassive pulmonary embolism. Vasc Med 2016; 21: 47-52.

9. Özkan M, Gündüz S, Biteker M, Astarcioglu MA, Çevik C, Kaynak E, et al. Comparison of different TEE-guided thrombolytic regimens for prosthetic valve thrombosis: theTROIA trial. JACC Cardiovasc Imaging 2013; 6: 206-216.

10. Zhang Z, Zhai ZG, Liang LR, Liu FF, Yang YH, Wang C. Lower dosage of recombinant tissue-type plasminogen activator (rt-PA) in the treatment of acute pulmonary embolism: a systematic review and meta-analysis. Thromb Res 2014; 133: 357-363.

11. Singh S, Houng A, Reed GL. Releasing the brakes on the fibrinolytic system in pulmonary emboli: Unique effects of plasminogen activation and $\alpha 2$-antiplasmin inactivation. Circulation 2017; 135: 1011-1020.

12. Sharifi M, Bay C, Skrocki L, Rahimi F, Mehdipour M, "MOPETT" Investigators. Moderate pulmonary embolism treated with thrombolysis (from the "MOPETT" Trial). Am J Cardiol 2013; 111: 273-277.

13. Kucher N, Boekstegers P, Müller OJ, Kupatt C, BeyerWestendorf J, Heitzer T, et al. Randomized, controlled trial of ultrasound-assisted catheter-directed thrombolysis for acuteintermediate-risk pulmonary embolism. Circulation 2014; 129: 479-486.

14. Ozcinar E, Cakici M, Dikmen Yaman N, Baran C, Aliyev $\mathrm{A}$, Inan $\mathrm{B}$, et al. Thrombus resolution and right ventricular functional recovery using ultrasound-accelerated thrombolysis in acute massive and submassive pulmonary embolism. Int Angiol 2017; 36: 428-437.

15. Meyer G, Vicaut E, Danays T, Agnelli G, Becattini C, BeyerWestendorf J, et al. Fibrinolysis for patients with intermediaterisk pulmonary embolism. NEnglJMed 2014; 370: 1402-1411. 